\title{
Indicadores de sustentabilidade energética: uma ferramenta de apoio à formulação de políticas energéticas mais sustentáveis
}

Políticas energéticas sustentáveis devem ser desenvolvidas com o intuito de contribuir para a sustentabilidade energética, o que envolve a garantia na geração transmissão, distribuição e utilização de energia para os múltiplos tipos de usuários. Os indicadores de energia são ferramentas que avaliam variáveis importantes do sistema de energia e que podem fornecer informações para apoiar tomada de decisões capazes de gerar políticas energéticas mais sustentáveis. Este artigo tem como objetivo propor um conjunto de indicadores de energia na perspectiva de dimensões específicas de sustentabilidade energética. Tais indicadores são úteis para o processo de formulação e implementação de políticas e planejamento energético para dado espaço territorial e escopo temporal. A proposição dos indicadores de sustentabilidade energética foi desenvolvida a partir de um estudo bibliométrico, para identificação de indicadores relacionados a: acesso à energia, eficiência energética e diversificação da matriz. Os indicadores do conjunto final foram alocados com base em três dimensões: acesso à energia, diversificação da matriz e eficiência energética. Tais indicadores apresentam definições, critérios e parâmetros contributivos para à formulação e implementação de políticas e planejamentos energéticos capazes de tornar o processo mais sustentável em contextos e contingências diversas.

\section{Indicators of energy sustainability: a tool to support the formulation of more sustainable energy policies}

\begin{abstract}
Sustainable energy policies must be developed with the aim of contributing to energy sustainability, which involves guaranteeing the generation, transmission, distribution and use of energy for the multiple types of users. Energy indicators are tools that assess important variables of the energy system and can provide information to support decision-making capable of generating more sustainable energy policies. This article aims to propose a set of energy indicators in the perspective of specific dimensions of energy sustainability. These indicators are useful for the process of formulation and implementation of policies and energy planning for a given territorial space and temporal scope. The proposal of the indicators of energy sustainability was developed from a bibliometric study, to identify indicators related to: access to energy, energy efficiency and matrix diversification. The indicators of the final set were allocated based on three dimensions: access to energy, matrix diversification and energy efficiency. These indicators present definitions, criteria and contributory parameters for the formulation and implementation of energy policies and plans capable of making the process more sustainable in diverse contexts and contingencies.
\end{abstract}

Keywords: Indicators; Energy; Sustainability; Energy Policy.

Topic: Desenvolvimento, Sustentabilidade e Meio Ambiente

Reviewed anonymously in the process of blind peer.
Received: $10 / 02 / 2019$

Approved: 25/03/2019
Joyce Aristércia Siqueira Soares

Universidade Federal de Campina Grande, Brasil

http://lattes.cnpq.br/2061306679679154

http://orcid.org/0000-0002-4648-6320

joycearistercia@gmail.com

Gesinaldo Ataíde Cândido (iD

Universidade Federal de Campina Grande, Brasil

http://lattes.cnpq.br/2771934125977891

http://orcid.org/0000-0002-3112-0254

gacandido@uol.com.br
Referencing this:

SOARES, J. A. S.; CÂNDIDO, G. A.. Indicadores de sustentabilidade energética: uma ferramenta de apoio à formulação de políticas energéticas mais sustentáveis. Revista Ibero Americana de Ciências Ambientais, v.10, n.2, p.284-303, 2019. DOI:

http://doi.org/10.6008/CBPC2179-6858.2019.002.0024 


\section{INTRODUÇÃO}

Tem ocorrido uma recente preocupação com a sustentabilidade energética no mundo, influenciada pela necessidade de garantir a segurança do aprovisionamento e fornecimento de energia bem como diminuir o aumento das temperaturas globais, associadas ao uso de combustíveis fósseis (ABBASI et al., 2011; OWUSU et al., 2016; DAVY et al., 2017). Os riscos em torno da garantia de segurança energética, atualmente, estão associados ao uso excessivo de combustíveis fósseis e matriz energética pouco diversificada e, portanto, de maior impacto ambiental, o que sujeita o sistema energético a possíveis crises energéticas e danos ambientais.

A crise energética decorre de dois fatores: padrão de fornecimento de energia e padrão de consumo (QINGCHUN, 2018). A falta de equilíbrio entre esses dois fatores resulta em não atendimento da demanda e busca de soluções, na maioria das vezes, caras e poluentes. Os danos ambientais se concretizam principalmente, quando há uma predominância de uso de fontes mais poluentes, que contribuem para o aumento das emissões de gases de efeito estufa e para um sistema energético injusto e inacessível, sobretudo para as populações mais pobres, que de acordo com Owusu et al. (2016) já chegam a 1,4 bilhão de pessoas, das quais, $86 \%$ vivem em áreas rurais.

Com relação às fontes poluentes, a preocupação central está voltada para as mudanças climáticas e os impactos relacionados ao uso excessivo de combustíveis fósseis e sua relação com o aumento das temperaturas globais (DAVY et al., 2017). Esta preocupação alerta para a necessidade de diversificação das matrizes energéticas, através da inserção de fontes de menor impacto ambiental e uso eficiente da energia. (OWUSU et al., 2016).

Com o intuito de responder a estas preocupações, os governos, em todas as partes do mundo, têm tentado formular políticas que conduzam seus sistemas de energia para garantir a segurança energética e adaptação às mudanças do clima, através da inserção gradual das energias renováveis ao longo do tempo (HADIAN et al., 2015). Contudo, a formulação de políticas energéticas mais sustentáveis não é tarefa fácil, uma vez que a sustentabilidade energética percebida ou diagnosticada pode variar em função do contexto e das próprias características do sistema energético. Isto, em parte, se deve ao fato de que pode acontecer de uma matriz energética ser predominantemente renovável e ainda assim não ser sustentável em termos de acesso aos serviços de energia, diversificação e uso eficiente, principalmente quando não existe uma distribuição equilibrada dos recursos energéticos nas diferentes regiões (QINGCHUN, 2018).

O Brasil, por exemplo, possui uma das matrizes mais sustentáveis do mundo, em função da predominância de recursos renováveis, com destaque para o recurso hidráulico. No entanto, o país já enfrentou crises energéticas que comprometeram o fornecimento seguro para atendimento da demanda de energia no país. Apesar da significância do recurso renovável, há ainda uma predominância do uso do recurso hidráulico para a geração de energia que é comprometido toda vez que o recurso se torna escasso, em função das próprias características físicas e climáticas do país (ROSA et al., 2003). 
Nesse caso, a política energética enquanto instrumento orientador das decisões, acerca da atuação do setor energético, deve considerar as características e o contexto em que cada sistema energético está inserido, requerendo planejamento, otimização e controle (OWUSU et al., 2016). Dentro dessa perspectiva, a fim de apoiar a formulação de políticas energéticas, os indicadores de energia são ferramentas que ajudam a medir variáveis importantes que devem ser consideradas no processo de formulação de políticas, uma vez que avaliam impactos atuais e tendências futuras do sistema de energia (VERA et al., 2007). Um indicador de energia é uma ferramenta que é utilizada para avaliar o desempenho de um sistema de energia, que ajuda a compreender as complexas interações que o sistema tem com aspectos econômicos, sociais e ambientais (NARULA et al., 2015).

Um conjunto de indicadores, enquanto ferramenta para apoiar a formulação de políticas energéticas mais sustentáveis, tem como função a medição de variáveis que influenciam o desempenho do sistema de energia, em termos ambientais, sociais e econômicos. Muito mais que isso, os indicadores precisam avaliar a sustentabilidade do sistema de energia em termos de acesso à energia, eficiência do sistema e impactos desse sistema ao meio ambiente relacionados a não diversificação da matriz.

O acesso à energia está relacionado ao acesso fiável e acessível a equipamentos de cozinha limpos, uma conexão à eletricidade e um aumento do consumo de eletricidade para atingir a média nacional (OECD, 2014). O acesso à energia é fundamental para promover o desenvolvimento e diminuir a disparidade entre o número de pessoas que ainda não têm acesso aos serviços básicos de energia de forma justa e segura (VERA et al., 2007; OWUSU et al., 2016).

A eficiência energética de acordo com Schlomann et al. (2015) é tipicamente definida a partir de um consumo físico de energia como insumo, que pode ser eletricidade, calor ou qualquer outra forma física de energia, que é comparada a um certo valor de uso, como saída, serviço, bem ou energia. A eficiência energética busca melhorar o uso das fontes de energia de forma racional e otimizada. No caso da diversificação da matriz está relacionado a inclinação para o uso de energias renováveis, promovendo a diversificação gradual, através da participação dessas fontes de energia,ao longo do tempo (HADIAN et al., 2015). Essas três dimensões estão diretamente relacionadas, uma vez que os esforços em eficiência energética, combinados com a expansão e geração de energias mais limpas, podem ajudar a garantir acesso confiável ao maior número de pessoas a um preço justo (CAN et al., 2018).

A partir destas considerações, o objetivo deste estudo foi propor um conjunto de indicadores de energia, alocados de acordo com as três dimensões da sustentabilidade energética, para apoiar o processo de formulação de políticas energéticas mais sustentáveis. Para atingir o objetivo proposto, foi realizada uma pesquisa bibliométrica para levantamento de indicadores de energia, especificamente, relacionados com as dimensões da sustentabilidade energética: diversificação da matriz energética, eficiência energética e acesso aos serviços de energia. O processo de identificação e seleção dos indicadores de energia foi realizado em quatro etapas: identificação dos indicadores em sites de instituições e em periódicos científicos, seleção de indicadores genéricos, comparação com os indicadores da Agência Internacional de Energia Atômica (IAEA) 
e a relação com as três dimensões da sustentabilidade energética, em termos de contribuição para a promoção de cada uma das dimensões.

Além deste conteúdo introdutório o artigo está assim estruturado: fundamentação teórica explorando os temas sustentabilidade energética e indicadores de energia. Em seguida, são apresentados os procedimentos metodológicos para o alcance do objetivo proposto, seguido da apresentação e análise dos resultados e as considerações finais.

\section{REVISÃO TEÓRICA}

\section{Sustentabilidade energética}

A energia tem relação direta com o conceito mais amplo de sustentabilidade e afeta a maior parte da sociedade (NARULA et al., 2015). Isso pode ser percebido pela relação de inserção dos recursos energéticos em praticamente todos os processos e atividades econômicas do mundo e em todos os setores, como insdustrial, transporte, residencial e comercial. Soma-se a isso o fato de que a obtenção dos recursos energéticos, renováveis ou não, é realizada a partir do ambiente e esse processo gera resíduos nas diferentes etapas de produção, transporte, armazenamento e utilização, que são liberados no ambiente e podem impactar negativamente as diferentes formas de vida no planeta (ROSEN, 2009).

A busca de uma condição de sustentabilidade energética é hoje uma necessidade que nações no mundo inteiro precisam alcançar em seus sistemas de energia e está diretamente relacionada com o impacto ambiental do uso da energia (NARULA et al., 2015). Essa necessidade surge em função da maneira insustentável com que os recursos energéticos são explorados e utilizados, causando problemas de ordem ambiental, econômica, social e, de acordo com Owusu et al. (2016) também resultam em conflitos geopolíticos, militares, além das flutuações nos preços dos combustíveis. Dentre esses problemas, a questão ambiental está relacionada ao aquecimento global e a necessidade de redução das emissões de Gases de Efeito Estufa (GEE) provenientes do uso de combustíveis fósseis (OWUSU et al., 2016).

Embora ainda não exista uma definição universal do termo sustentabilidade energética, ela deve ser entendida como a aplicação das definições gerais de sustentabilidade à energia (ROSEN, 2009; NARULA et al., 2015). De acordo com Rosen (2009) a sustentabilidade energética está relacionada à prestação de serviços de energia de forma sustentável, de modo que todas as pessoas tenham acesso ao fornecimento desse serviço para o atendimento de suas necessidades básicas, agora e futuramente, sem que isso gere impactos ambientais. Narula et al. (2015) também tratam da sustentabilidade energética a partir da definição de desenvolvimento sustentável.

Lior (2008) apresenta uma definição de sustentabilidade energética relacionada com as atividades que envolvem o uso e a produção de energia, de modo que essas atividades sejam sustentáveis. De acordo com este autor quando tais atividades são sustentáveis, significa que elas atendem as necessidades presentes sem comprometer a capacidade das futuras gerações terem suas necessidades atendidas, de forma harmônica entre necessidades econômicas, sociais e ambientais (LIOR, 2008). 
As três definições, Lior (2008), Rosen (2009) e Narula et al. (2015), decorrem do conceito geral de desenvolvimento sustentável expresso no Relatório Brundtland, em 1987, quando definiu o desenvolvimento sustentável como aquele capaz de atender as necessidades das gerações presentes sem comprometer as gerações futuras de também terem suas necessidades atendidas.

Na literatura, outros trabalhos, como de Campos (2005), Baltelo (2008), Lior (2008), Neves et al. (2010), Rosen (2009; 2012) e Narula et al. (2015), tratam da sustentabilidade energética, sob diferentes perspectivas, porém sempre apresentando a relação da energia e de como ele é explorada e utilizada e sua implicação com a sustentabilidade. Não apenas como recurso econômico estratégico, mas como fator preponderante para o desenvolvimento social e econômico de uma sociedade, a energia passa a ser alvo de discussões em torno da sustentabilidade.

Neves et al. (2010) abordam a sustentabilidade energética dentro de uma perspectiva local, em que propõem uma estrutura de indicadores locais de sustentabilidade energética para apoiar planos e ações mais sustentáveis, que considerem, sobretudo, as alterações climáticas. Baltelo (2008) apresenta uma perspectiva da sustentabilidade energética a partir da análise das políticas energéticas direcionadas para a implementação de fontes de menor impacto ambiental, com destaque para o Programa de Incentivo às Fontes Alternativas de Energia (PROINFA), desenvolvido e implementado no Brasil, bem como apresenta experiências internacionais também relacionadas ao desenvolvimento de políticas para inserção de fontes renováveis. Por fim, Campos (2005) analisa a sustentabilidade energética numa perspectiva das ações das empresas do setor elétrico brasileiro, a partir da proposição de indicadores que forneçam relatórios de sustentabilidade desse setor.

Enquanto Neves et al. (2010) e Campos (2005), propõem ferramentas para avaliar a sustentabilidade energética e Baltelo (2008), Narula et al. (2015)apontam para o desenvolvimento de políticas de incentivo às renováveis, Rosen (2012) alerta para a abrangência da sustentabilidade energética que não deve ser entendida apenas como a inserção de fontes consideradas sustentáveis, já que o sistema de energia está relacionado, para além do uso sustentável dessas fontes, com o uso de tecnologias de aproveitamento de energia, de conversão em formas de utilização, transporte e armazenamento de energia.

Em outras palavras, a inserção de fontes de energias de menor impacto ambiental por si só não garante sustentabilidade para o sistema de energia, é necessário o correto aproveitamento e uso dessas fontes, o que está relacionado com a aplicação de instrumentos de eficiência energética que resultem em conservação de energia (NARULA et al., 2015). Acrescenta-se o fato de que o alcance de um sistema de energia sustentável também deve considerar o acesso aos serviços de energia de forma segura e justa a toda população. Isto está relacionado ao fato de que ainda existem mais de 1,4 bilhão de pessoas no mundo sem acesso aos serviços de eletricidade (OWUSU et al., 2016). A esse respeito, Vera et al. (2007) alertam que a prestação de serviços energéticos confiáveis, seguros e ambientalmente corretos para atendimento das necessidades de desenvolvimento econômico e social é fundamental para o alcance do desenvolvimento sustentável. 
Assim, diante do exposto e das perspectivas de análise da sustentabilidade energética consideradas por Baltelo, (2008), Campos (2005), Lior (2008), Neves et al. (2010) e Rosen (2009; 2012) e da relação que o sistema energético tem com a sustentabilidade, a análise da sustentabilidade energética se assenta sob três dimensões: Acesso à energia, eficiência energética e diversificação da matriz. Essas três dimensões estão diretamente relacionadas, uma vez que os esforços em eficiência energética, combinados com a expansão e geração de energias mais limpas, podem ajudar a garantir acesso confiável ao maior número de pessoas (CAN et al., 2018).

A promoção da eficiência energética está relacionada a instrumentos que incentivem o uso eficiente e a conservação de energia. De acordo com a International Energy Agency (IEA), a eficiência energética é necessária para garantir segurança energética, acessível e sustentável para as gerações futuras. Além do mais, é um recurso disponível para todas as nações e é o caminho menos oneroso para enfrentar os obstáculos relacionados com a segurança energética, as questões ambientais e econômicas (IEA, 2017). Nessa perspectiva, quando a IEA afirma que a eficiência energética é um recurso disponível para todos, ela se refere ao fato de que todos os governos podem tomar iniciativas para otimizar o uso de energia, que assegure disponibilidade de recursos energéticos para atendimento das demandas da sociedade.

Com relação à diversificação da matriz energética, pode-se incluir tanto fontes de origem fóssil como fontes menos poluentes, como biomassa, energia proveniente dos ventos, das marés, fotovoltaica que, embora também gerem resíduos e perturbações durante todo o processo de geração de energia, são consideradas menos impactantes que as de origem fóssil e, portanto, são mais preferíveis e mais sustentáveis. Muitos países em todo o mundo têm vindo a desenvolver políticas para tentar preservar a sua segurança energética nacional e para se adaptar às mudanças climáticas decorrentes das emissões de gases de efeito estufa. Assim, políticas de diversificação da matriz energética estão voltadas para o uso de energias renováveis, com a participação dessas fontes de energia aumentando gradualmente ao longo do tempo (HADIAN et al., 2015).

O acesso à energia é fundamental para a erradicação da pobreza e para a melhoria da qualidade dos padrões vida e bem-estar (OTTINGER, 2005). De acordo com Mensah et al. (2014), o acesso aos serviços modernos de energia tem sido mencionado como essencial para o desenvolvimento. Vera et al. (2007) já discorriam sobre a importância da garantia de acesso aos serviços de energia como fator limitador ou impulsionador do desenvolvimento sustentável. Isso acontece porque a distribuição dos serviços de energia não acontece de forma homogênea e contribui para que muitas pessoas no mundo não tenham acesso aos serviços mínimos de energia a preços acessíveis (OWUSU et al., 2016).

Nessa perspectiva, os indicadores de energia têm sido desenvolvidos com o intuito de apoiar o processo de formulação de políticas energéticas em todo o mundo (HI, 2011; INDICATORS, 2014; KEIRSTEAD, 2007; KRUYT et al., 2009; OLADE, 2000; OTTINGER, 2005; PATLITZIANAS et al., 2008; WCE, 2016). Os indicadores são ferramentas de coleta de informações que podem ajudar no monitoramento dos sistemas de energia para apoiar a formulação de políticas energéticas mais sustentáveis. Desse modo, a partir da discussão sobre sustentabilidade energética e suas três dimensões: acesso à energia, eficiência energética e 
diversificação da matriz, um conjunto de indicadores capaz de diagnosticar a sustentabilidade de uma sistema de energia, precisa identificar informações relacionada com iniciativas de programas de eficiência energética, inserção de fontes de menor impacto ambiental ou informações relacionadas às emissões de GEE e ao mesmo tempo proporcionar informações sobre a evolução do acesso aos serviços básicos de energia, conforme Figura 1.

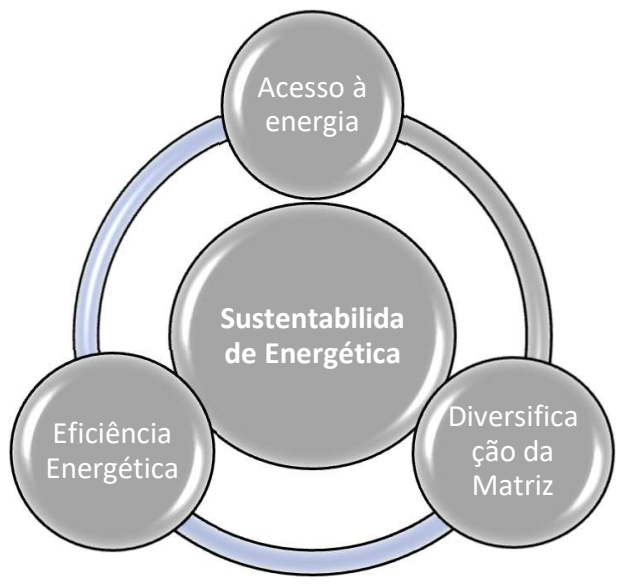

Figura 1: Pilares da Sustentabilidade Energética.

Contudo, se faz necessário, buscar indicadores de energia que possam traduzir esse conjunto de informações a partir dessas três dimensões. Assim, é preciso selecionar indicadores de energia que contribuam para o acesso aos serviços de energia, que contribuam para diversificação da matriz energética e diminuição de impactos ambientais e que incentivem o desenvolvimento e implementação de programas de eficiência energética. Portanto, tais indicadores deverão ser alocados em função de critérios de promoção de cada uma das dimensões da sustentabilidade energética, conforme será apresentado mais à frente.

\section{Indicadores de Energia}

De acordo com a OECD (1993), um indicador é um parâmetro ou um valor resultante de parâmetros que indica e fornece informações acerca de um fenômeno, com o intuito de entender melhor a complexa realidade que envolve a ciência, a sociedade, a tecnologia, a economia e o meio ambiente (PATLITZIANAS et al., 2008). A esse respeito, Narula et al. (2015) definem indicador de energia como uma ferramenta que é utilizada para avaliar o desempenho de um sistema de energia.

Com relação aos indicadores de sustentabilidade, estes têm se mostrado importantes ferramentas de medição de efeitos atuais e futuros de determinados sistemas sobre o meio ambiente natural e a sociedade. No contexto da sustentabilidade energética, indicadores de energia devem servir como ferramentas para avaliar e monitorar as implicações que determinadas ações dentro de um sistema de energia têm para o desenvolvimento sustentável em termos de impactos ambientais, econômicos e sociais (IAEA, 2005).

Para Vera et al. (2007), indicadores de energia não devem ser entendidos apenas como estatísticas de energia, mas como um conjunto de informações que proporcionam um entendimento mais profundo das relações causais entre a energia, o meio ambiente e a economia, capazes de comunicar informação de forma 
simplificada da realidade de um fenômeno. Além do mais, os indicadores devem ter relevância política e dar suporte ao processo de tomada de decisão (BELLEN, 2005).

A primeira iniciativa para o desenvolvimento de indicadores de energia foi desenvolvida pela Agência Internacional de Energia Atômica (IAEA) em parceria com o Departamento de assuntos Econômicos e Sociais das Nações Unidas (UNDESA), a Agência Internacional de Energia (AIE) e outras organizações internacionais, como o Gabinete de estatísticas da União Europeia - EUROSTAT, em 2001, e apresentada na nona sessão da Comissão sobre Desenvolvimento Sustentável (CSD-9). As principais questões que direcionaram a construção desses indicadores foram à acessibilidade de energia, eficiência energética, energia renovável, tecnologias de combustíveis fósseis avançadas, tecnologias de energia nuclear, energia rural e energia de transportes. 0 resultado foi a proposição de um conjunto de 30 indicadores de energia para o desenvolvimento sustentável, classificados em três principais dimensões: social, econômica e ambiental. Esses indicadores foram, em seguida, aplicados no Brasil, Cuba, Lituânia, México, Rússia, Eslováquia e Tailândia, com o intuito de apoiar as políticas energéticas dessas nações, bem como identificar as prioridades e objetivos de acordo com a necessidade energética para o desenvolvimento sustentável (OECD, 2014).

Apesar de significar um avanço para avaliação das questões energéticas, para efeito do presente trabalho, considerou-se que esse conjunto de indicadores de energia da IAEA é muito genérico e global. Embora permita avaliar a sustentabilidade energética de nações e fornecer informações sobre a situação do sistema de energia, quando se deseja avaliar um sistema energético mais específico, estes indicadores apresentam limitações que impedem a visualização de questões mais pontuais que determinados sistemas de energia têm, sendo necessário fazer adaptações, inserindo ou retirando indicadores que se adéquem a realidade do fenômeno estudado, ou seja, que considerem o contexto e as características do sistema de energia, como é o caso de nações que possuem matrizes energéticas predominantemente renováveis, como é o caso do Brasil, por exemplo.

Assim, a partir do entendimento de que é necessário um conjunto de indicadores que considere as características e peculiaridades de cada sistema de energia, um conjunto de indicadores baseados nos pilares da sustentabilidade energética, quais sejam, diversificação da matriz energética, eficiência energética e acesso à energia, poderão representar melhor a realidade do sistema energético em termos de avaliação da efetividade das políticas energéticas desenvolvidas e implementadas, de modo a fornecer um diagnóstico que considere a proposta da sustentabilidade energética.

\section{METODOLOGIA}

Quanto aos objetivos a pesquisa realizada pode ser caracterizada como exploratória. Quanto aos procedimentos técnicos se caracteriza como pesquisa bibliográfica e documental. Com relação à abordagem do problema, se caracteriza como bibliométrica, uma vez que no processo de identificação dos indicadores de energia, fez-se um levantamento quantitativo em sites de instituições nacionais e internacionais, bem como de plataformas de periódicos científicos para identificar autores e trabalhos sobre indicadores de energia e especificamente indicadores que trouxessem relação com a sustentabilidade energética e, 
especificamente, relacionados com as dimensões da sustentabilidade energética: diversificação da matriz energética, eficiência energética e acesso aos serviços de energia.

A partir da identificação e seleção dos documentos e respectivos conjuntos de indicadores, a proposição do conjunto de indicadores de sustentabilidade energética englobou 4 etapas: identificação e seleção de indicadores de energia, filtragem dos indicadores selecionados para deixar no conjunto geral apenas indicadores genéricos, comparação do conjunto geral com o conjunto da IAEA, já que este conjunto de indicadores de energia é o mais consolidado em termos de indicadores de energia para o desenvolvimento sustetável e, por último, fez-se a relação dos indicadores com as três dimensões da sustentabilidade energética, a partir de critérios de como cada indicador pode contribuir para a promoção dessas dimensões.

A primeira etapa consistiu na identificação e seleção de conjuntos de indicares na literatura e sites institucionais, (HI, 2011; INDICATORS, 2014; KEIRSTEAD, 2007; KRUYT et al., 2009; OLADE, 2000; OECD, 2014; PATLITZIANAS et al., 2008; WCE, 2016). Nessa primeira etapa partiu-se de uma pesquisa bibliométrica que, de acordo com Maia (2015) dos processos de comunicação escrita, tratamento quantitativo das propriedades e comportamentos das informações registradas.

Um dos objetivos da pesquisa bibliométrica é buscar identificar tendências e bases de crescimento de conhecimentos em determinada área científica. De acordo com Soares (2018), a bibliometria toma por alguns indicadores bibliométricos. Os três mais comuns estão relacionados à lei de Lotka, que mede a produtividade de autores e identifica centros de pesquisa, a lei de Zipf que mede a frequência de determinadas palavras de acordo com a sua relevância em um determinado texto e a lei de Bradford, que mede a produtividade de periódicos em determinada área de conhecimento. Esta etapa se caracteriza pela aplicação da lei de Lotka e Zipf, uma vez que a busca dos indicadores de energia objetivou identificar instituições e autores em bases de pesquisas com base em palavras-chaves específicas. Assim, essa etapa pode ser representada pela Figura 2.

A segunda etapa consistiu na seleção de indicadores genéricos. Os indicadores genéricos foram considerados aqueles que avaliam questões gerais do sistema energético, como por exemplo, o indicador eficiência energética na indústria pode ser representado pelo indicador genérico 'eficiência energética', apenas. $O$ indicador eficiência energética já abrange todos os setores econômicos. Essa seleção foi feita a partir da percepção do pesquisador, em um processo de identificação de indicadores específicos que poderiam ser representados por indicadores genéricos.

A terceira etapa consistiu em um processo de comparação entre os indicadores, com o intutito de identificar indicadores semelhantes em termos de definição e avaliação. Optou-se pelo conjunto da AIEA, como conjunto base, para desenvolver o processo de comparação, de modo que, a medida que indicadores se apresentavam semelhantes ou iguais, esses eram excluídos do conjunto geral, resultando em um conjunto de 48 indicadores. 


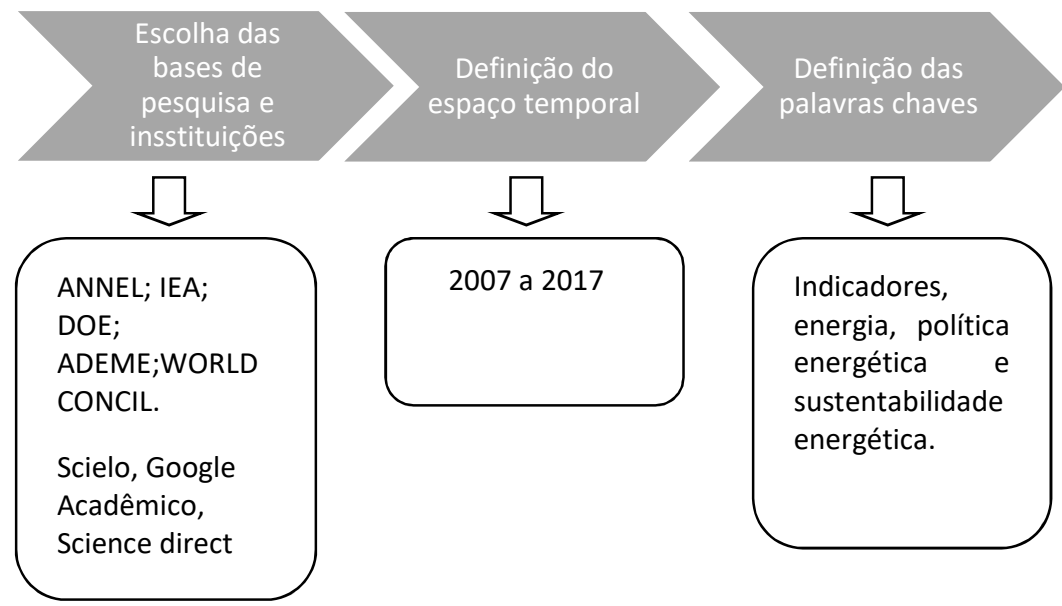

Figura 2: Processo de identificação dos indicadores.

Por fim, a quarta etapa desenvolveu-se a partir de uma análise, com base na percepção do pesquisador, relacionando o conjunto de 48 indicadores de energia com as dimensões da sustentabilidade energética, como diversificação da matriz energética, eficiência energética e acesso aos serviços de energia. Foram escolhidos e alocados indicadores que pudessem contribuir para a promoção de cada uma das dimensões da sustentabilidade: acesso à energia, eficiência energética e diversificação da matriz. Os indicadores identificados como não contributivos para a promoção dessas dimensões foram excluídos do conjunto geral. Após a execução das quatros etapas para a identificação e seleção dos indicadores de energia, foi proposto um conjunto de 17 indicadores de sustentabilidade energética para apoiar a implementação de políticas energéticas mais sustentáveis.

\section{RESULTADOS E DISCUSSÃO}

\section{Etapa 1}

Esta etapa consistiu na identificação e seleção de indicadores de energia, a partir de um estudo bibliométrico. Após, a identificação e seleção dos conjuntos de indicadores, com base nos objetivos propostos por cada conjunto de indicadores, foram selecionadas 4 instituições internacionais e 3 autores com seus respectivos conjuntos de indicadores de energia, conforme Tabela 1.

Tabela 1: Indicadores de Energia.

\begin{tabular}{|l|l|l|l|}
\hline Indicadores de Energia & Objetivo & Autor/Instituição & $\begin{array}{l}\text { Quantidade de } \\
\text { Indicadores }\end{array}$ \\
\hline $\begin{array}{l}\text { Indicadores de energia para o } \\
\text { desenvolvimento sustentável }\end{array}$ & Apoiar a política energética & (IAEA, 2005) & 30 \\
\hline $\begin{array}{l}\text { Indicadores de energia para a } \\
\text { Sustentabilidade }\end{array}$ & $\begin{array}{l}\text { Monitoramento de variáveis que } \\
\text { influenciam o desenvolvimento sustentável }\end{array}$ & (OLADE, 2000) & 08 \\
\hline Indicadores de Eficiência Energética & $\begin{array}{l}\text { Analisar as tendências de eficiência } \\
\text { energética mundial e proporcionar } \\
\text { subsídios para os decisores políticos }\end{array}$ & (WCE, 2016) & 41 \\
\hline $\begin{array}{l}\text { Indicadores de Eficiência Energética } \\
\text { envolvem as atividades econômicas e } \\
\text { humanas, o consumo de energia e as } \\
\text { emissões de CO } 2\end{array}$ & (OECD, 2014) & 38 \\
\hline $\begin{array}{l}\text { Indicadores de Processamento de } \\
\text { Informação de Política Energética } \\
\text { propício para o Ecodesenvolvimento }\end{array}$ & $\begin{array}{l}\text { Formulação de políticas energéticas mais } \\
\text { sustentáveis }\end{array}$ & (HI, 2011) & 24 \\
\hline
\end{tabular}




\begin{tabular}{|c|c|c|c|}
\hline Indicadores de Política Energética & $\begin{array}{l}\text { Apoiar os formuladores de políticas e } \\
\text { tomadores de decisão, no sentido de } \\
\text { construir políticas energéticas sustentáveis }\end{array}$ & $\begin{array}{l}\text { (PATLITZIANAS et } \\
\text { al., 2008) }\end{array}$ & 28 \\
\hline $\begin{array}{l}\text { Indicadores de sustentabilidade } \\
\text { energética para sistemas urbanos }\end{array}$ & Avaliar a sustentabilidade urbana & (KEIRSTEAD, 2007) & 42 \\
\hline Indicadores de Segurança Energética & Avaliar a segurança energética & (KRUYT et al., 2009) & 08 \\
\hline
\end{tabular}

O trabalho desenvolvido pela AIEA apresenta um conjunto de indicadores voltados para avaliar a sustentabilidade de forma geral de um sistema de energia (IAEA, 2005). É um conjunto genérico, desenvolvido com esta finalidade. Além do mais, esse conjunto de indicadores expressa claramente quais indicadores estão relacionados com cada dimensão da sustentabilidade. Apesar de ser um conjunto mais completo, o seu caráter genérico não permite que um sistema de energia específico, como é o caso dos sistemas renováveis, seja avaliado de forma precisa, sendo necessária a retirada de alguns indicadores, bem como a inserção de outros.

Os indicadores apresentados pela Olade (2000), também trazem informações genéricas acerca de um sistema de energia. Estão relacionados a um conjunto de aspectos que influenciam o sistema energético em diferentes níveis de desenvolvimento e sustentabilidade e que estão relacionados com riscos e vulnerabilidades que impedem o desenvolvimento socioeconômico, fornecimento de energia justo e impacto sobre o meio ambiente.

Os indicadores expressos pela OECD tratam de um conjunto de indicadores mais específicos. Estão relacionados com a questão da eficiência energética e foram desenvolvidos com a finalidade de apoiar analistas e formuladores de políticas no desenvolvimento de indicadores de eficiência energética (OECD, 2014). Ele analisa cada setor econômico de forma individualizada, fornecendo uma metodologia de análise das tendências de consumo de energia. Apesar de ter sido uma escolha inicial, este conjunto foi excluído do conjunto final de indicadores gerados pelo presente trabalho pelo fato de expressarem informações específicas de setores econômicos que são apresentadas por indicadores genéricos identificados em outros conjuntos.

O conjunto de indicadores da HI (2011) consiste em uma ferramenta para utilização de informações prontamente disponíveis para tomada de decisão mais coerente relacionada com a questão energética. Sua finalidade é analisar o quanto a política energética nacional contribui em termos de desenvolvimento sustentável. Assim como os indicadores da AIEA, também levam em consideração as dimensões da sustentabilidade, além da dimensão tecnológica, governança, vulnerabilidade e resiliência. Os indicadores apresentados pelo Conselho Mundial de Energia consistem em uma ferramenta de monitoramento das políticas de governo para o setor energético relacionadas às tendências de eficiência energética a nível mundial, bem como as tendências do uso de energia e as emissões de $\mathrm{CO}_{2}$ (WCE, 2016).

O trabalho dos três autores selecionados Keirstead (2007), Kruyt et al. (2009) e Patlitzianas et al. (2008) são conjuntos de indicadores de energia mais específicos, tendo em vista que abordam questões voltadas para a segurança energética, política energética e sustentabilidade de sistemas de energia urbano. Os indicadores apresentados por Keirstead (2007) é o resultado de uma seleção de um conjunto de 
indicadores a partir de outros trabalhos para identificar aqueles que melhor expressam a sustentabilidade urbana de forma geral. Como estão voltados para sistemas de energia urbanos, esse conjunto foi selecionado para análise no presente trabalho, com base no entendimento de que o ambiente urbano está inserido em um sistema de energia maior que precisa ser considerado na análise da sustentabilidade energética.

Já os indicadores apresentados por Patlitzianas et al. (2008) foram desenvolvidos com a finalidade de apoiar os decisores políticos, analistas e cidadãos para o desenvolvimento de uma política energética sustentável. Por fim, o trabalho de Kruyt et al. (2009), apresenta um conjunto de indicadores relacionados com a segurança do fornecimento de energia a longo prazo, considerando quatro dimensões: disponibilidade, acessibilidade e aceitabilidade da energia. É um conjunto de indicadores de segurança energética voltado para sistemas energéticos de fonte fóssil e traz uma análise acerca do petróleo direcionada para o contexto no qual esta fonte está inserida.

Esses conjuntos de indicadores expostos na Tabela 1 são indicadores relacionados com a energia ou algum aspecto importantante para análise da sustentabilidade. Cada um, dentro das suas características e objetivo de cada autor ou intituição, são ferramentas de análise da situação energética de um país ou de um sistema isolado de energia, para fornecimento de diagnóstico que possa indicar problemas ou ajustes necessários em termos de política energética.

A reunião desses oito trabalhos, resultaram em um conjunto unificado de 228 indicadores de energia que serviram de base para a proposição de um quadro de indicadores de sustentabilidade energética para apoiar a formulação de políticas energéticas mais sustentáveis (HI, 2011; OECD, 2014; KEIRSTEAD, 2007; KRUYT et al., 2009; OLADE, 2000; IAEA, 2005; PATLITZIANAS et al., 2008; WCE, 2016).

\section{Etapa 2}

Na segunda etapa foi realizada seleção dos indicadores genéricos. Essa seleção foi feita a partir da percepção do pesquisador, em um processo de identificação de indicadores específicos que poderiam ser representados por indicadores genéricos, conforme Figura 3. Aqui, entendeu-se que os indicadores genéricos já abordavam os indicadores específicos. Nesta etapa observou-se que o conjunto de indicadores da OECD era composto por indicadores muito específicos, de modo que optou-se por excluí-lo do conjunto final (OECD, 2014). Nesse processo foram excluídos 165 indicadores restando no conjunto geral 63 indicadores de energia.

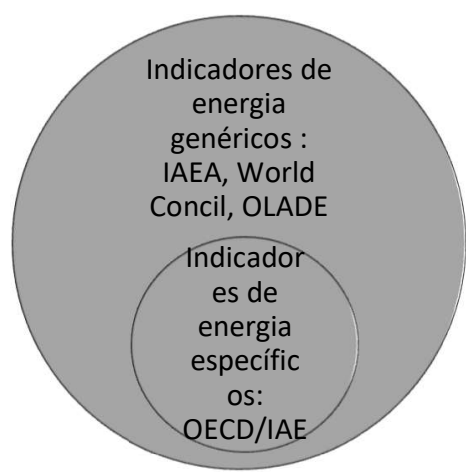

Figura 3: Filtragem dos indicadores genéricos. 


\section{Etapa 3}

A terceira etapa consistiu em um processo de comparação entre o conjunto geral de indicadores resultantes com o intutito de identificar indicadores iguais ou com definições semelhantes. Vale salientar que optou-se pelo conjunto da IAEA como base de comparação, tendo em vista ser o conjunto considerado mais completo e consolidado em termos de processo formulação e pesquisas que citam esses indicadores. 0 processo de comparação pode ser analisado na Figura 4. Após a comparação entre os indicadores obteve-se um conjunto único de 48 indicadores de energia.

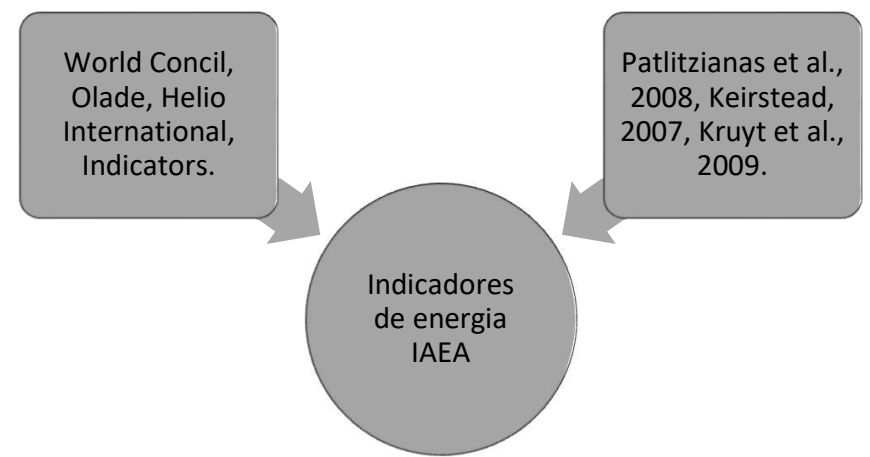

Figura 4: Processo de comparação dos indicadores.

\section{Etapa 4}

Na quarta etapa realizou-se uma análise, com base na percepção do pesquisador, relacionando o conjunto de 48 indicadores de energia com as dimensões da sustentabilidade energética, como diversificação da matriz energética, eficiência energética e acesso aos serviços de energia, que resultaram em um conjunto de 17 indicadores. Nesta etapa tomou-se como critério para a escolha e alocação desses indicadores a definição de cada uma dessas dimensões e quais indicadores poderiam contribuir para a promoção dos mesmos, conforme Figura 5 que apresenta esse processo. Os indicadores foram selecionados com base na sua capacidade para promover o acesso à energia e medir o nível de acesso da população aos serviços básicos de energia, conforme Tabela 2 .

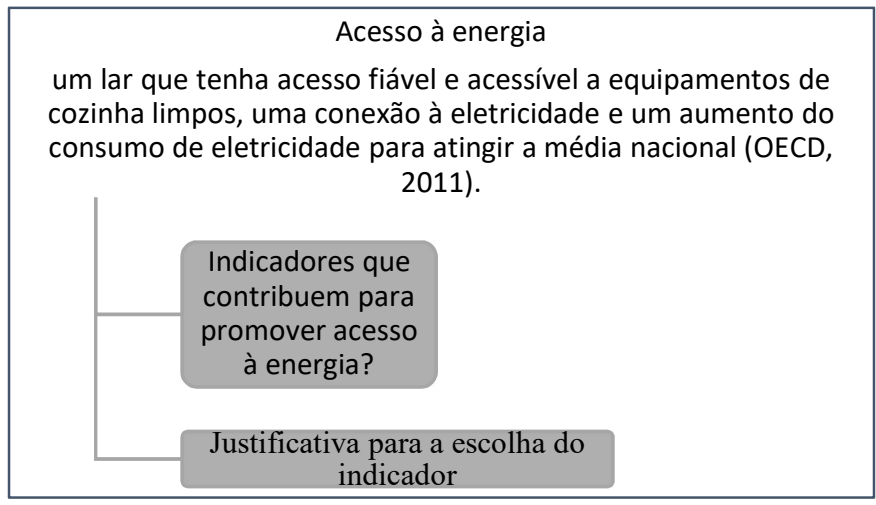

Figura 5: Dimensão Acesso à energia.

O indicador \% de família ou população com acesso a eletricidade ou dependentes de energias não comerciais está relacionado a percentagem de famílias ou população sem acesso a serviços energéticos comerciais incluindo a electricidade, ou fortemente dependentes de opções de energia 'tradicionais' não 
comerciais, tais como lenha, resíduos de colheita e esterco de animais (IAEA, 2007). A seleção desse indicador para a sustentabilidade energética justifica-se pelo fato de que a prestação e o acesso seguro e adequado aos serviços comerciais de energia são fundamentais para uma alimentação adequada, habitação, água, saneamento, educação, saúde e comunicação, tendo em vista que esses serviços contribuem positivamente para o desenvolvimento econômico e para a diminuição das desigualdades e, portanto para o desenvolvimento sustentável.

Tabela 2: Indicadores que promovem Acesso à energia.

\begin{tabular}{|l|}
\hline$\%$ de família ou população com acesso a eletricidade ou dependentes de energias não comerciais. \\
\hline \% da renda familiar gasta com combustível ou eletricidade. \\
\hline Consumo de energia per capita \\
\hline Emprego \\
\hline
\end{tabular}

O indicador \% da renda familiar gasta com combustível ou eletricidade está relacionado com o rendimento disponível das famílias (ou consumo privado) gasto em combustível e eletricidade (IAEA, 2007). Como impulsionadora do desenvolvimento sustentável a variável renda, deve proporcionar condições mínimas de acesso à energia, sobretudo para as populações menos favorecidas, de modo que a variável renda pode ser um indicador que contribui ou inibe o acesso aos serviços de energia.

O indicador consumo per capita de energia está relacionado ao uso de energia em termos de oferta total de energia primária (OTE), o consumo final total (CFT) e utilização final de electricidade per capita (IAEA, 2007). Esse indicador é fundamental para mensurar o progresso da qualidade de vida tendo em vista que a energia é um fator limitador ou impulsionador do desenvolvimento econômico. Por outro lado, o aumento do consumo de energia pode trazer uma pressão maior sobre o meio ambiente através da exploração dos recursos energéticos, sendo portanto, importante a mensuração do consumo per capita para avaliar os impactos positivos e negativos que esse consumo pode proporcionar.

O indicador Emprego está relacionado ao nível de emprego da população ou região. Este indicador tem relação direta com o indicador renda, tendo em vista que pode promover as condições de renda mínima para que a população tenha acesso aos serviços de energia. A escolha desse indicador deve-se ao fato de que o nível de emprego de um país reflete diretamente na melhoria das condições de vida da população através da geração de emprego e renda. O mesmo processo de seleção e justificativa de escolha de indicadores foi realizado para a dimensão Eficiência energética, conforme Figura 6.

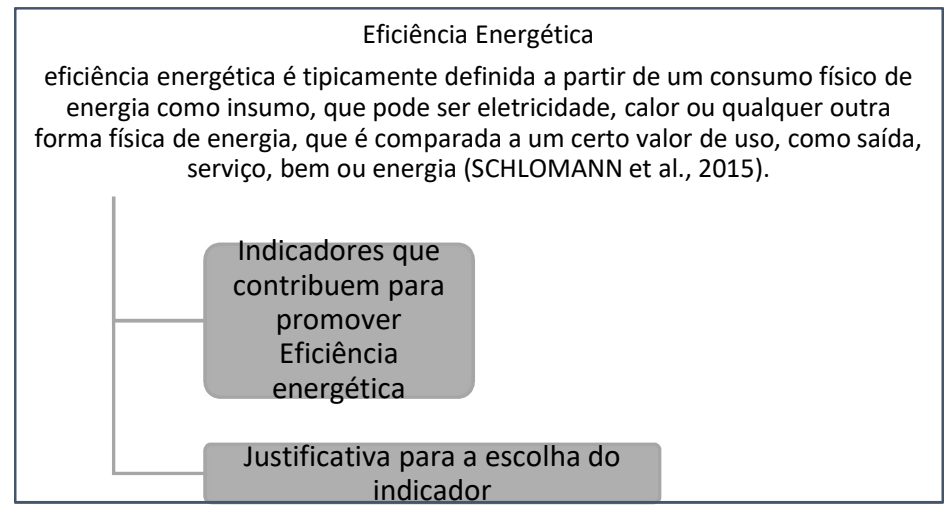

Figura 6: Dimensão Eficiência Energética. 
De acordo com Schlomann et al. (2015) todas as discussões aprofundadas sobre eficiência energética e objetivos de eficiência energética partem de um valor específico do consumo de energia em unidades físicas. Ambos os índices, produção por entrada de energia e entrada de energia por produto, podem servir como indicadores de eficiência energética. Os indicadores selecionados a partir dessa dimensão da sustentabilidade energética podem ser observados na Tabela 3.

Tabela 3: Indicadores para a Eficiência Energética.

\begin{tabular}{|l|}
\hline $\begin{array}{l}\text { Intensidade energética (por unidade do PIB). } \\
\text { Intensidade energética industrial }\end{array}$ \\
\hline Intensidades energéticas agrícolas \\
\hline Intensidade energética no comércio e setor de serviços \\
\hline Intensidadeenergética nas famílias \\
\hline Investimento no setor de energia (P\&D e estoque de capital). \\
\hline Gestão de crises
\end{tabular}

O primeiro indicador selecionado foi intensidade energética e junto a ele os indicadores de intensidade energética industrial, agrícola, no comércio e setor de transporte e intensidade energética nas famílias. A intensidade energética (por unidade de PIB) corresponde a taxa da oferta total de energia primária (OIE), o consumo final total (CFT) e uso de eletricidade para o produto interno bruto (PIB) (IAEA, 2007). A intensidade energética industrial corresponde ao uso de energia por unidade de valor agregado no setor industrial e por setores selecionados de energia intensiva; A intensidade energética agrícola se refere ao uso final de energia por unidade de valor agregado agrícola; A intensidade energética no comércio e setor de transportes corresponde ao uso final de energia por unidade de serviço e valor agregado comercial e; a intensidade energética nas famílias corresponde a quantidade de uso de energia por uso final residencial por pessoa ou casa ou unidade de área de chão, ou por eletrodoméstico (IAEA, 2007).

A escolha desses indicadores justifica-se pelo fato de que há uma necessidade de melhoria na eficiência energética nestes setores, de modo a melhorar de forma mais efetiva a utilização dos recursos energéticos e a redução dos impactos ambientais negativos (IAEA, 2007). O intutuito é reduzir o consumo de energia e as emissões de gases de efeito estufa, conforme apresenta Schlomann et al. (2015), nesses setores, através de programas de eficiência energética.

Outro indicador selecionado para essa dimensão foi o investimento no setor de energia (P\&D e estoque de capital) que corresponde a percentagem da riqueza líquida de um país destinada ao setor de energia. A importância desse indicador está diretamente relacionado a destinação de recursos para o desenvolvimento de programas e tecnologias de promoção a redução do uso de energia e melhoria da eficiência energética.

O indicador Gestão de crises está relacionado a existência de programas alternativos em situações de crise, como o não atendimento da demanda pelos serviços de energia. Este indicador é importante, principalmente para garantir que em situações de crise, existam meios de contornar os problemas e garantir acesso contínuo dos serviços de energia sem prejudicar os que dela dependem. 0 mesmo processo de seleção e justificativa de escolha de indicadores foi realizado para a dimensão Diversificação da Matriz 
Energética, conforme Figura 7. Os indicadores selecionados para compor essa dimensão estão relacionados na Tabela 4.

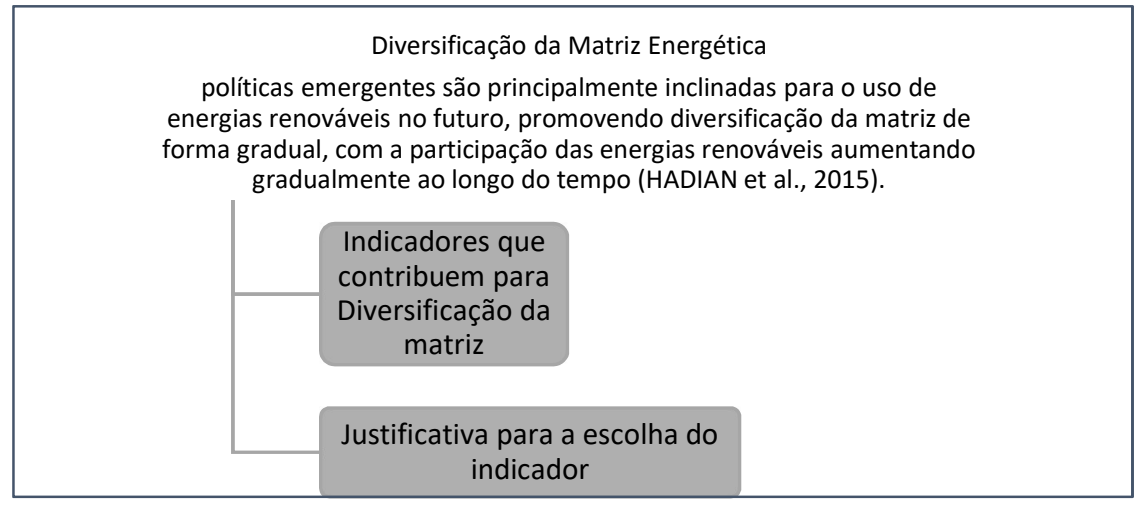

Figura 7: Dimensão Diversificação da Matriz Energética.

Tabela 4: Dimensão Diversificação da Matriz Energética.

\begin{tabular}{|l|}
\hline Percentual de geração de energia elétrica proveniente de fontes não emissoras de carbono \\
\hline Geração de energia elétrica de fontes renováveis \\
\hline Emissões de gases de efeito estufa (GEE) na produção e no uso de energia por unidade de PIB. \\
\hline Concentração de poluentes no ambiente e em áreas urbanas. \\
\hline Poluição do ar através da geração de energia \\
\hline Taxa de desmatamento atribuída a geração de energia \\
\hline
\end{tabular}

O indicador percentual de geração de energia elétrica proveniente de fontes não emissoras de carbono corresponde a participação das fontes de energia sem carbono no fornecimento de energia primária e na geração de eletricidade e na capacidade de geração (IAEA, 2007). A justificativa para a escolha desse indicador deve-se ao fato de que o uso de fontes não fósseis é essencial para a proteção ambiental, segurança energética e diversificação da matriz energética e corrobora com Hadian et al. (2015 ) acerca da necessidade de inserção gradual de fontes de menor impacto ambiental na matriz energética.

O segundo indicador geração de energia elétrica por fontes renováveis está relacionado à participação das energias renováveis no fornecimento total de energia primária, no consumo final total e na geração de eletricidade e na capacidade de geração por fontes renováveis (IAEA, 2007). A introdução de fontes renováveis contribui para a segurança do aprovisionamento energético e diminuição dos impactos ambientais negativos.

O indicador emissões de gases de efeito estufa (GEE) na produção e no uso de energia por unidade de PIB corresponde às emissões de gases de efeito estufa (GEE) a partir da produção e uso de energia, per capita e por unidade de produto interno bruto (PIB), incluindo dióxido de carbono $\left(\mathrm{CO}_{2}\right)$, metano $\left(\mathrm{CH}_{4}\right)$ e óxido nitroso $\left(\mathrm{N}_{2} \mathrm{O}\right)$ (IAEA, 2007); Já o indicador concentração de poluentes no ambiente e em áreas urbanas corresponde as concentrações ambientais de poluentes atmosféricos, como ozônio, monóxido de carbono, partículas (PM10, PM2.5, partículas suspensas totais [TSP], fumaça preta), dióxido de enxofre, dióxido de nitrogênio, benzeno e chumbo (IAEA, 2007); O indicador poluição do ar através da geração de energia se refere às emissões de poluentes atmosféricos de todas as atividades relacionadas à energia, incluindo produção e transporte de eletricidade (IAEA, 2007). Esses indicadores foram selecionados devido a crescente preocupação com as maiores concentrações de poluentes no ar, decorrentes da emissão de gases de efeito estufa, associados a produção e geração de energia. A concentração de poluentes é amplamente influenciada 
pelos padrões de produção e consumo de energia, que, por sua vez, são afetados pela intensidade e eficiência energética (IAEA, 2007).

O indicador taxa de desmatamento atribuída a geração de energia corresponde a mudança anual na quantidade de área de floresta natural e de plantação rastreada ao longo do tempo que poderia ser atribuída ao uso de madeira como combustível para fins energéticos (IAEA, 2007). A justificativa para a escolha desse indicador deve-se ao fato de que as florestas são ecossistemas fundamentais para o desenvolvimento sustentável, uma vez que desenvolvem papeis de proteção do solo, da água e filtragem de poluentes. Este indicador aponta para a avaliação do quanto a matriz energética pode ser responsável pelo desmatamento e emissão de gases de efeito estufa, provenientes da queima de madeira como combustível para geração de energia.

Por fim, esse processo de seleção e alocação de indicadores de energia com base nas dimensões da sustentabilidade energética, resultaram em um conjunto de 17 indicadores de energia para apoiar a formulação de políticas energéticas mais sustentáveis, conforme Tabela 5. Em cada uma das dimensões, acesso à energia, eficiência energética e diversificação da matriz, estão indicadores que podem servir de referência para avaliar cada uma dessas dimensões e apontar necessidades de políticas energéticas que garantam acesso seguro, justo, a preços acessíveis, aos serviços básicos de energia para populações menos favorecidas e que ao mesmo tempo possam contribuir com o crescimento econômico e desenvolvimento econômico e social e a preservaçãao do meio ambiente.

Os indicadores relacionados com o pilar acesso aos serviços de energia foram aqueles que pudessem avaliar o retorno das políticas energéticas em termos de beneficios para a sociedade, especificamente com questões relacionadas com a garantia de acesso mínimo aos serviços de eletricidade. $\mathrm{O}$ acesso à energia contribui com a erradição da pobreza e eleva os padrões de vida refletindo diretamente no desenvolvimento de países (MENSAH et al., 2014). Esses indicadores devem refletir o número de pessoas e condições de acesso a preços justos aos serviços básicos de energia, tendo em vista que ainda existem muitas pessoas sem acesso mínimo a esses serviços acessíveis (OWUSU et al., 2016).

Para o segundo pilar da sustentabilidade energética, eficiência energética, buscou-se relacionar indicadores que expressassem informações sobre uso de energia em setores preponderantes para o crescimento econômico e desenvolvimento social. Assim, os indicadores selecionados a partir desse pilar foram indicadores como: intensidade energética em setores como o industrial, agrícola, de serviços e comércio e residencial (famílias).

Tabela 5: Indicadores de Energia.

\begin{tabular}{|l|}
\hline Indicadores de Sustentabilidade Energética \\
\hline Dimensão 1 - Acesso à Energia \\
\hline \% de família ou população com acesso a eletricidade ou dependentes de energias não comerciais. \\
\hline \% da renda familiar gasta com combustível ou eletricidade. \\
\hline Consumo de energia per capita \\
\hline Emprego \\
\hline Dimensão 2 - Eficiência Energética \\
\hline $\begin{array}{l}\text { Intensidade energética (por unidade do PIB). } \\
\text { Intensidade energética industrial }\end{array}$ \\
\hline Intensidades energéticas agrícolas \\
\hline
\end{tabular}




\begin{tabular}{|l|}
\hline Intensidade energética no comércio e setor de serviços \\
\hline Intensidadeenergética nas famílias \\
\hline Investimento no setor de energia (P\&D e estoque de capital). \\
\hline Gestão de crises \\
\hline Dimensão 3 - Diversificação da Matriz \\
\hline Percentual de geração de energia elétrica proveniente de fontes não emissoras de carbono \\
\hline Geração de energia elétrica de fontes renováveis \\
\hline Emissões de gases de efeito estufa (GEE) na produção e no uso de energia por unidade de PIB. \\
\hline Concentração de poluentes no ambiente e em áreas urbanas. \\
\hline Poluição do ar através da geração de energia \\
\hline Taxa de desmatamento atribuída a geração de energia \\
\hline
\end{tabular}

Por fim, o terceiro pilar da sustentabilidade, Diversificação da matriz, optou-se por indicadores que pudessem avaliar variáveis que refletissem o quanto uma matriz energética pode ser considerada diversificada mediante a inserção de fontes de menor impacto ambiental como por exemplo: "Participação de fontes não emissoras de carbono na matriz; geração de energia por fontes renováveis ou ainda emissão de gases de efeito estufa, concentação de poluentes" e assim por diante. É necessário o correto aproveitamento dessas fontes no sistema de energia através de políticas que incentivem a gradual transição de fontes baseadas no carbono por fontes mais limpas que possam contribuir com a redução das emisões de GEE (NARULA et al., 2015; HADIAN et al., 2015).

\section{CONCLUSÕES}

A necessidade de desenvolver políticas energéticas mais sustentáveis tem se mostrado cada vez mais urgente em função da relação que a prestação de serviços de energia de forma justa, segura e confiável têm com o desenvolvimento sustentável, uma vez que o acesso aos serviçso de energia pode se constituir como um fator limitador ou impulsionador desse tipo de desenvolvimento (VERA et al., 2007). Assim, as políticas energéticas precisam ser desenvolvidas dentro de uma perspectiva de sustentabilidade energética, o que pressupõe políticas orientadas para promover acesso aos serviços de energia, diversificação da matriz energética e eficiência energética.

O desenvolvimento dessas políticas mais sustentáveis podem ser melhor desempenhado através do uso de ferramentas, que dêem indicações acerca de variáveis importantes de energia e que informem os tomadores de decisão acerca de quais os melhores cursos de ação a serem seguidos, dentro de uma perspectiva de sustentabilidade energética. Assim, os indicadores de energia, enquanto ferramentas de medição de impactos e tendências atuais e futuras dos sistemas de energia, podem desenvolver esse papel de apoio à formulação dessas políticas.

O estudo em questão objetivou propor um conjunto de indicadores de sustentabilidade energética para apoiar a formulação de políticas energéticas mais sustentáveis e, resultou em um conjunto de 17 indicadores de energia, alocados a partir das dimensões da sustentabilidade energética e selecionados a partir de critérios de promoção dessas dimensões. Neste estudo, fez-se uma seleção de indicadores a partir da literatura, HI (2011), OECD (2014), Keirstead (2007), Kruyt et al. (2009), OLADE (2000), IAEA (2005), Patlitzianas et al. (2008) e WCE (2016) e, após quatro etapas, obteve-se o conjunto final. 
Os indicadores do conjunto final foram alocados com base em três dimensões: acesso a energia, diversificação da matriz e eficiência energética, tendo em vista serem estes os três pilares da sustentabilidade energética. Desse modo, os indicadores selecionados apresentaram variáveis e informações que pudessem contribuir para a promoção de cada uma dessas dimensões, com o intutito de propor um conjunto que pudesse ser caracterizado como indicadores de sustentabilidade energética.

Por fim, o conjunto final de indicadores de sustentabilidade energética se constitui em uma ferramenta de orientação para tomada de decisão, relacionadas com a energia, dentro de uma perspectiva de sustentabilidade. Acredita-se que, para cada realidade o conjunto proposto pode sofrer alterações, seja através da insersão ou retirada de indicadores com o intuito de aproxima-lo da realidade estudada.

Como limitações do conjunto apresentado, pode-se mencionar o fato de ainda não ter sido aplicado, o que impede a formulação de possíveis inferências sobre o mesmo. Contudo, enquanto ferramenta de medição de variáveis de energia pode ser adaptado para cada realidade que se deseja estudar a partir do momento em que é utilizado para avaliar ações de políticas e programas de energia.

\section{REFERÊNCIAS}

ABBASI, T.; ABBASI, S. A. Renewable energy sources: Their impact on global warming and pollution. PHI Learning Pvt. Ltd., 2011.

BALTELO, R.. A caminho da sustentabilidade energética: como desenvolver um mercado de renováveis no Brasil. 2008.

BELLEN, H. M. V.. Indicadores de sustentabilidade: uma análise comparativa. In: Indicadores de sustentabilidade: uma análise comparativa. Rio de Janeiro: FGV, 2005.

BORGES, F. Q.. Indicadores de sustentabilidade para a energia elétrica no estado do Pará. Revista Brasileira de Energia, v.15, n.2, p.119-151, 2009.

CAN, S. D. L. R., PUDLEINER, D., E PIELLI, K. Energy efficiency as a means to expand energy access: A Uganda roadmap. Energy Policy, v. 120, p. 354-364, 2018.

CAMPOS, J. J. F.. Sustentabilidade Energética no Brasil: proposta de indicadores para elaboração de relatórios de sustentabilidade por empresas do Setor Elétrico. Dissertação (Mestrado em Administração) - Fundação Getúlio Vargas, São Paulo, 2005.

COUNCIL, W. E.. Energy efficiency policies around the world: review and evaluation. London: WEC, 2008.

DAVY, R.; GNATIUK, N.; PETTERSSON, L.; BOBYLEV, L. Climate change impacts on wind energy potential in the European domain with a focus on the Black Sea. Renewable and Sustainable Energy Reviews, v.81, p.1652-1659, 2017.

HADIAN, S.; MADANI, K.. A system of systems approach to energy sustainability assessment: Are all renewables really green?. Ecological Indicators, v.52, p.194-206, 2015.

HI. Helio International. Processing Information for Energy Policies Conducive to Ecodevelopment. Ottawa, 2011.
IAEA. International Atomic Energy Agency. Energy indicators for sustainable development: guidelines and methodologies. Vienna: IAEA; 2005.

KEIRSTEAD, J.. Selecting sustainability indicators for urban energy systems. In: International Conference on Whole Life Urban Sustainability and its Assessment Glasgow. 2007.

KRUYT, B.; VUUREN, D. P.; VRIES, H. J.; GROENENBERG, H. Indicators for energy security. Energy Policy, v.37, n.6, p.2166-2181, 2009.

LIOR, N.. Energy resources and use: the present situation and possible paths to the future. Energy, v.33, n.6, p.842$857,2008$.

MAIA, J. L.; SERIO, L. C.; ALVES FILHO, A. G.. Pesquisa bibliométrica em estratégia como prática: resultados exploratórios e comparação de fontes. Sistemas \& Gestão, v.10, n.4, p.654-69, 2016.

MENSAH, G. S.; KEMAUSUOR, F.; BREW-HAMMOND, A. Energy access indicators and trends in Ghana. Renewable and Sustainable Energy Reviews, v.30, p.317-323, 2014

NARULA, K.; REDDY, B. S.. Three blind men and an elephant: The case of energy indices to measure energy security and energy sustainability. Energy, v.80, p.148-158, 2015.

NEVES, A. R.; LEAL, V.. Energy sustainability indicators for local energy planning: review of current practices and derivation of a new framework. Renewable and Sustainable Energy Reviews, v.14, n.9, p.2723-2735, 2010.

OECD. Core set of indicators for environmental performance reviews. Environment Monographs. Paris, 1993. 
OECD. Indicadores de Eficiência Energética: essenciais para o desenvolvimento de políticas. OECD, 2014.

OWUSU, P.; ASUMADU-SARKODIE, S.. A review of renewable energy sources, sustainability issues and climate change mitigation. Cogent Engineering, v.3, n.1, 2016.

PATLITZIANAS, K. D.; DOUKAS, H.; KAGIANNAS, A. G.; E PSARRAS, J.. Sustainable energy policy indicators: Review and recommendations. Renewable Energy, v.33, n.5, p.966973, 2008.

QINGCHUN, Y.. A Study of Management System Based on Energy Crisis. In: IOP CONFERENCE SERIES: EARTH AND ENVIRONMENTAL SCIENCE. Anais. IOP Publishing, 2018.

ROSEN, M. A.. Energy sustainability: a pragmatic approach and illustrations. Sustainability, v.1, n.1, p.55-80, 2009.
ROSEN, M. A.. Engineering sustainability: A technical approach to sustainability. Sustainability, v.4, n.9, p.22702292, 2012.

SCHLOMANN, B.; ROHDE, C.; PLÖTZ, P.. Dimensions of energy efficiency in a political context. Energy Efficiency, v.8, n.1, p.97-115, 2015.

SOARES, S. V.; PICOLLI, I. R. A.; CASAGRANDE, J. L.. Pesquisa Bibliográfica, Pesquisa Bibliométrica, Artigo de Revisão e Ensaio Teórico em Administração e Contabilidade. Administração: Ensino e Pesquisa, v.19, n.2, p.1-19, 2018.

VERA, I.; LANGLOIS, L.. Energy indicators for sustainable development. Energy, v.32, n.6, p.875-882, 2007.

WCE. World Concil Energy. Indicadores de Eficiência Energética. Enerdata, 2016. 